\title{
Uncommon indications for associating liver partition and portal vein ligation for staged hepatectomy: a systematic review
}

\author{
Quirino Lai, Gianluca Mennini, Zoe Larghi Laureiro, Massimo Rossi \\ Hepatobiliary Surgery and Organ Transplantation Unit, Department of General Surgery and Organ Transplantation, Sapienza University of Rome, \\ Rome, Italy \\ Contributions: (I) Conception and design: Q Lai; (II) Administrative support: M Rossi; (III) Provision of study materials or patients: Q Lai; (IV) \\ Collection and assembly of data: Q Lai, Z Larghi Laureiro; (V) Data analysis and interpretation: Q Lai, G Mennini, Z Larghi Laureiro; (VI) \\ Manuscript writing: All authors; (VII) Final approval of manuscript: All authors. \\ Correspondence to: Quirino Lai, MD, PhD. Department of General Surgery and Organ Transplantation, Sapienza University of Rome, Viale del \\ Policlinico 155, 00161 Rome, Italy. Email: lai.quirino@libero.it.
}

\begin{abstract}
Associating liver partition and portal vein ligation for staged hepatectomy (ALPPS) represents an innovative surgical technique used for the treatment of large hepatic lesions at high risk for post-resection liver failure due to a small future liver remnant. The most significant amount of literature concerns the use of ALPPS for the treatment of hepatocellular carcinoma (HCC), cholangiocarcinoma (CCC), and colorectal liver metastases (CRLM). On the opposite, few is known about the role of ALPPS for the treatment of uncommon liver pathologies. The objective of the present study was to evaluate the current literature on this topic. A systematic review was performed according to the Preferred Reporting Items for Systematic Reviews and Meta-Analyses (PRISMA) guidelines. Eligible articles published up to February 2020 were included using the MEDLINE, Scopus, and Cochrane databases. Among the 486 articles screened, 45 papers met the inclusion criteria, with 136 described cases of ALPPS for rare indications. These 136 cases were reported in 18 different countries. Only in two countries, namely Germany and Brazil, more than ten cases were observed. As for the ALPPS indications, we reported 41 (30.1\%) cases of neuroendocrine tumor (NET) metastases, followed by 27 (19.9\%) cases of gallbladder cancer (GBC), nine (6.6\%) pediatric cases, six (4.4\%) gastrointestinal stromal tumors, six (4.4\%) adult cases of benign primary liver disease, four (2.9\%) adult cases of malignant primary liver disease, and 43 (31.6\%) adult cases of malignant secondary liver disease. According to the International ALPPS Registry data, less than 10\% of the ALPPS procedures have been performed for the treatment of uncommon liver pathologies. NET and GBC are the unique pathologies with acceptable numerosity. ALPPS for NET appears to be a safe procedure, with satisfactory long-term results. On the opposite, the results observed for the treatment of GBC are poor. However, these data should be considered with caution. The rationale for treating benign pathologies with ALPPS appears to be weak. No definitive response should be given for all the other pathologies. Multicenter studies are needed with the intent to clarify the potentially beneficial effect of ALPPS for their treatment.
\end{abstract}

Keywords: Neuroendocrine tumor (NET); gastrointestinal stromal tumors (GIST); gallbladder cancer (GBC); hepatoblastoma, metastases

Submitted Mar 08, 2020. Accepted for publication May 07, 2020.

doi: $10.21037 / \mathrm{hbsn}-20-355$

View this article at: http://dx.doi.org/10.21037/hbsn-20-355 


\section{Introduction}

Associating liver partition and portal vein ligation for staged hepatectomy (ALPPS) represents an innovative surgical technique used for the management of large hepatic lesions at high risk for post-resection liver failure due to a small future liver remnant (FLR) (1). Since its introduction in 2012 (2), ALPPS procedure has been considered, together with the well-known two-stage hepatectomy (TSH), as a useful tool for surgeons approaching extreme hepatic resections.

ALPPS has the peculiarity to reduce the time between the two stages of the hepatectomy when compared with the classic TSH. Consequently, this kind of procedure has determined through years a great interest among surgeons. In 2014, an international ALPPS Registry was founded (3), and several studies have been published considering its pros and cons (4).

The most significant amount of literature concerns the use of ALPPS for the treatment of hepatocellular carcinoma (HCC) $(5,6)$, cholangiocarcinoma (CCC) (7), and colorectal liver metastases (CRLM) $(8,9)$. On the opposite, few is known about the role of ALPPS for the treatment of uncommon liver pathologies.

Several aspects should justify a study focused on this aspect. First, no studies exist reporting the numerical impact of uncommon liver pathologies in terms of global ALPPS activity. Second, more robust experiences, although sparse, exist on some uncommon liver pathologies, justifying to investigate the impact of ALPPS on their treatment. Last, a study based on poorly investigated pathologies should represent a stimulus for the scientific community to improve the research on this specific field, consenting to construct multicenter experiences.

Consequently, the objective of the present systematic review was to investigate the role of ALPPS for the cure of uncommon liver pathologies.

\section{Methods}

\section{Search sources and study design}

A systematic review of the published literature focused on the clinical impact of ALPPS for the management of rare liver pathologies was undertaken. The search strategy was performed by the Preferred Reporting Items for Systemic Reviews and Meta-Analysis (PRISMA) guidelines, as well as PRISMA for abstracts (10).

A search of the MEDLINE, Scopus and Cochrane
Database was conducted using the following terms: (((“ALPPS”(Title/Abstract) OR “associating liver partition and portal vein ligation for staged hepatectomy"(Title/ Abstract)) OR “in situ split" (Title/Abstract)) OR "in situ splitting"(Title/Abstract)) OR "liver partition”(Title/Abstract) AND (“2012/01/01”(PDAT) : “2020/02/01"(PDAT)).

The systematic qualitative review included a priori search criteria of journal articles among adult (age $\geq 18$ years) human patients; studies were limited to the English language. Published reports were excluded in the following cases: (I) data on animal models; (II) studies focused on ALPPS for the treatment of CRLM, HCC, and CCC; (III) lacked sufficient clinical details; (V) included non-primary source data (e.g., review articles, non-clinical studies, letters to the editor, expert opinions, conference summaries).

Studies originating from the same center were analyzed, and possible overlapping of clinical cases was taken into account. Due to the limited number of extensive studies published in the literature on this field, case reports were included in the analysis.

\section{Data extraction and definitions}

Following a review of the full-texts from eligible studies, two independent authors (QL and ZLL) performed the data extraction and crosschecked all outcomes. During the selection of articles and extraction of the data, potential discrepancies were resolved with the consensus of a third reviewer (GM). Data were collected on the first author of the publication, year of publication, affiliation, country, study period, number of reported cases, patient age in years, gender, primary disease, tumor size, number of lesions, primary lesion location, neoadjuvant treatment, type of liver resection, mini-invasive liver surgery, days between first and second stage, FLR before the first stage (in $\mathrm{mL}$ or \%), FLR before the second stage (in $\mathrm{mL}$ or \%), FLR hypertrophy between the first-second stage (in \%), Dindo-Clavien grade after the second stage, length of stay after the second stage, 30-day mortality, adjuvant treatment, overall survival, and recurrence.

\section{Quality assessment and statistical analysis}

Selected studies were reviewed according to the representativeness of the study population, adequate evaluation of outcomes, sufficient length and adequacy of 


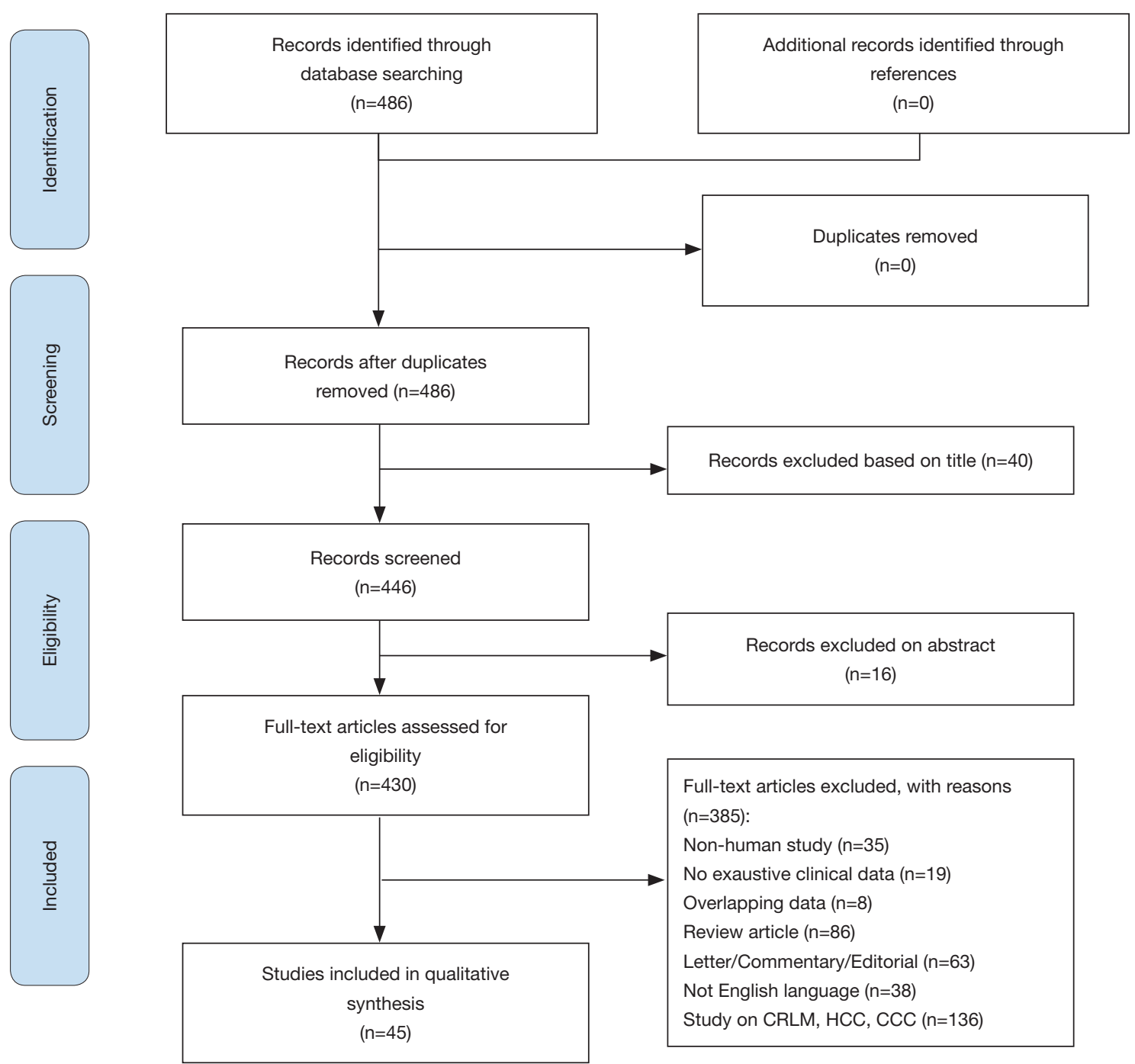

Figure 1 PRISMA flowchart of the literature search and study selection.

follow-up, and source of study funding. The quality of the papers was assessed using the Newcastle-Ottawa Quality Assessment Scale (NOS): studies with scores $>6$ were defined as high-quality studies (11).

Continuous variables were reported as medians and ranges. Categorical variables were noted as numbers and proportions. Statistical analyses were performed using the SPSS statistical package version 24.0 (SPSS Inc., Chicago, IL, USA).

\section{Results}

The PRISMA flow diagram schematically depicts the article selection process (Figure 1).
Among the 486 articles screened, 45 papers met the inclusion criteria (Table 1). The vast majority of the reported studies were composed of case reports or case series. In studies presenting a control group, no comparison between series with uncommon liver pathologies treated with ALPPS $v s$. other surgical approaches was performed. In turn, it was not possible to determine a NOS value in all the studies.

In eight studies, an evident data overlapping was reported. However, in many cases, it was impossible to determine if an overlapping was also present among the 45 selected studies, mainly in the case of papers coming from international databases $(14,21,22,24,36,45,47)$.

One hundred thirty-six ALPPS cases with rare 


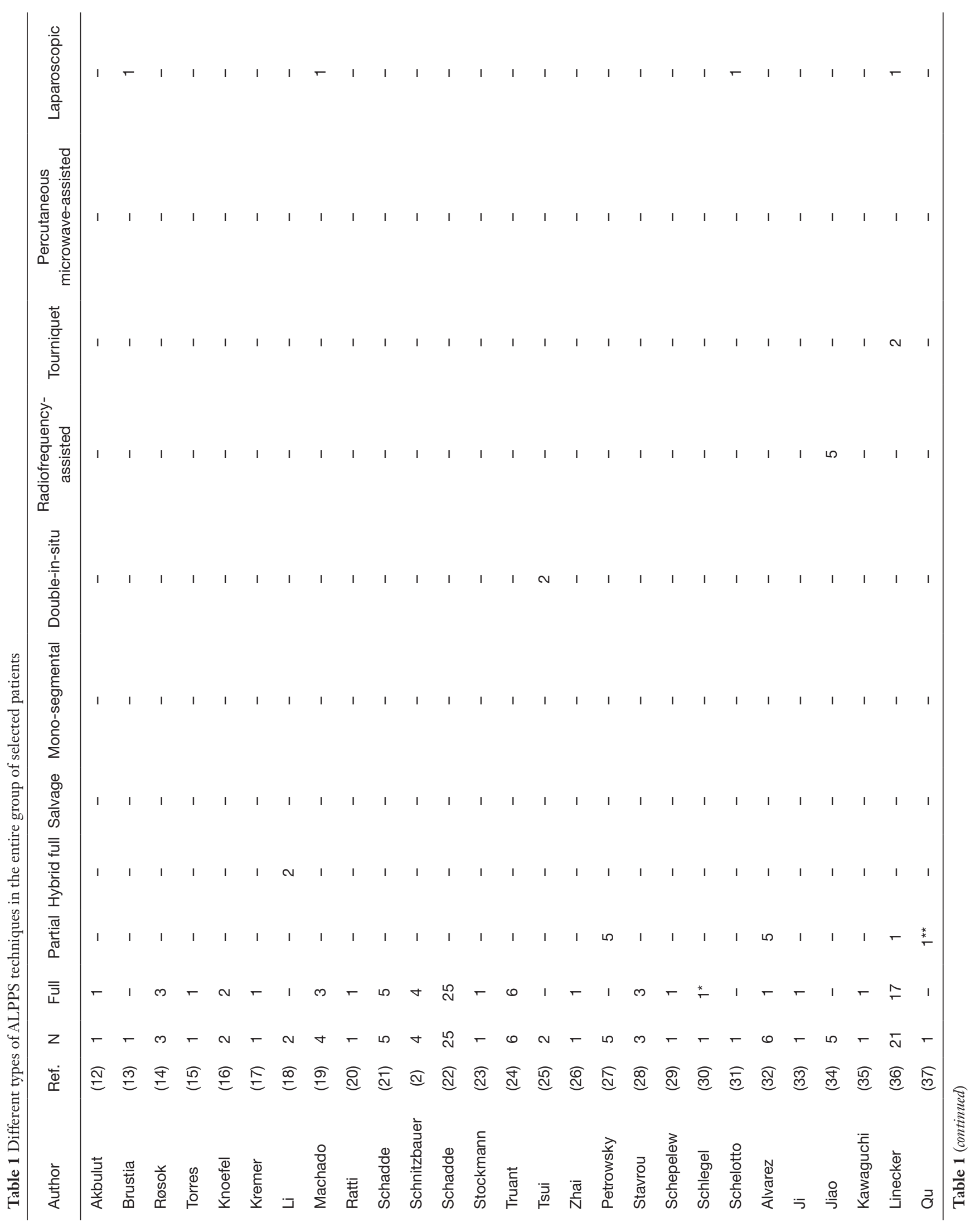




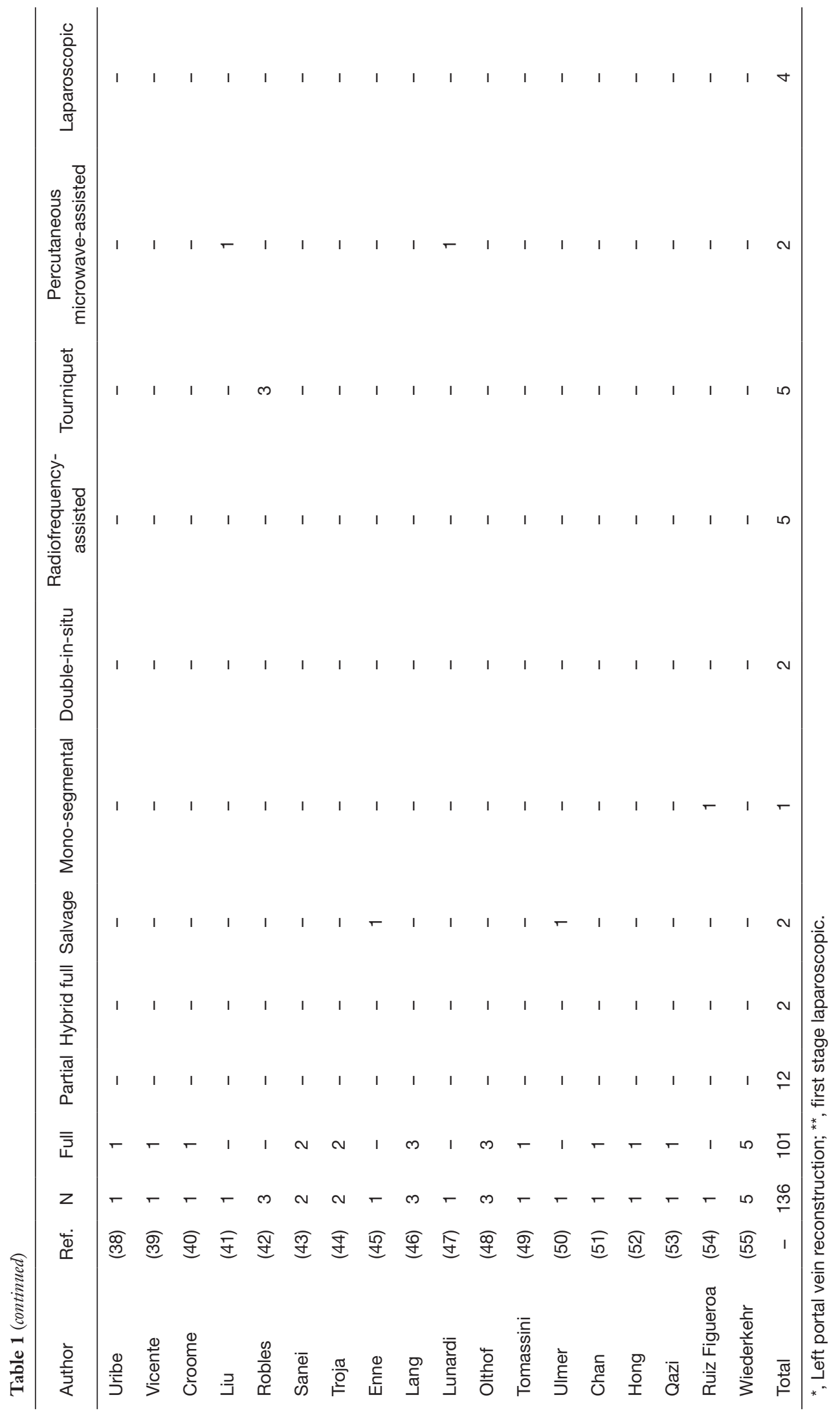




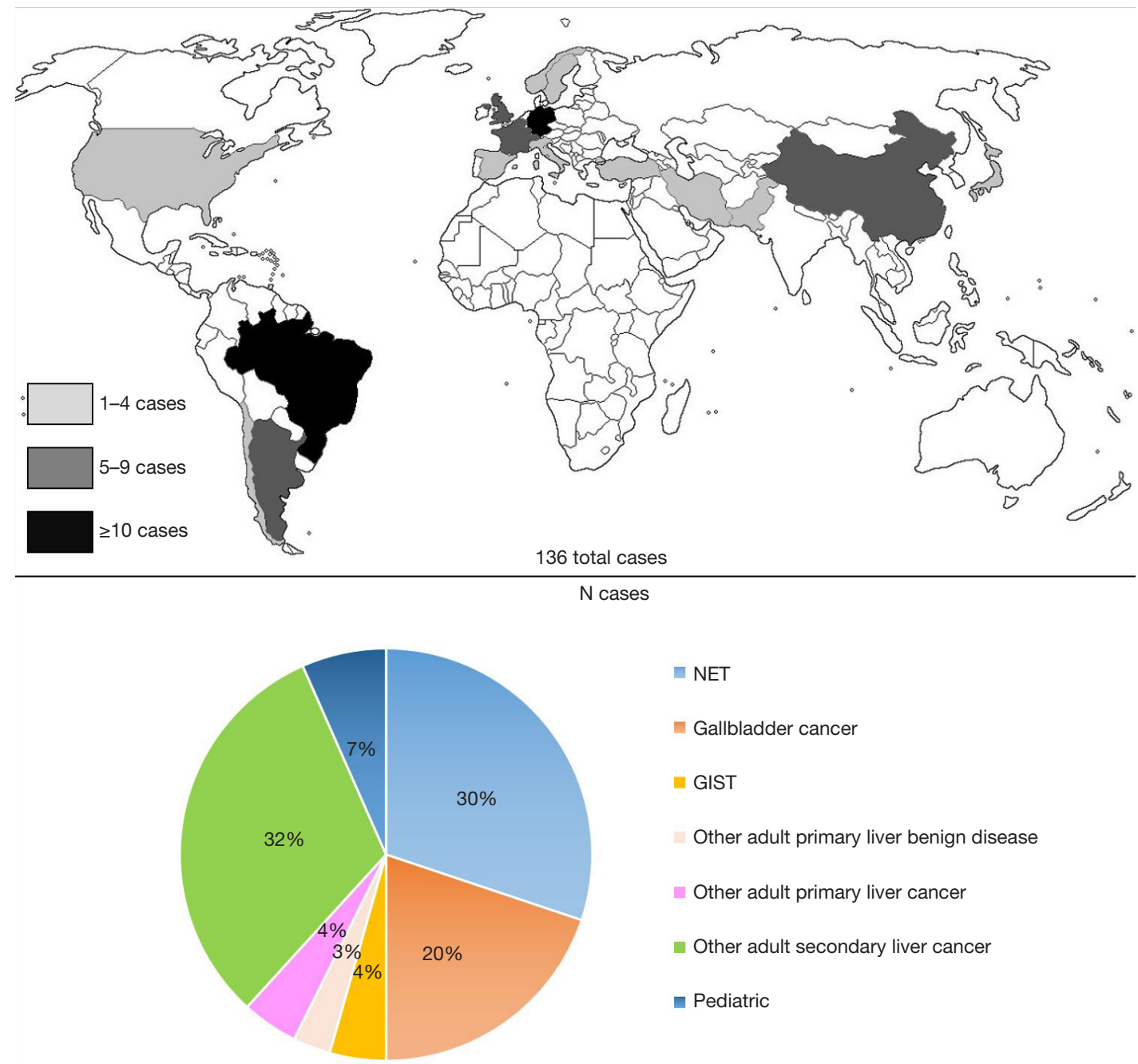

Figure 2 ALPPS cases for the treatment of uncommon liver pathologies detailed for geographical distribution and liver pathology.

indications were reported in the selected 45 studies (Figure 2). These 136 cases were reported in 18 different countries. Only in two countries, namely Germany and Brazil, more than ten cases were observed. In seven studies, data came from international registries, and, therefore, it was impossible to evaluate the country of provenience $(14,21,22,24,36,45,47)$.

As for the indication for ALPPS, we reported 41 (30.1\%) cases of neuroendocrine tumor (NET) metastases, followed by $27(20.0 \%)$ cases of gallbladder cancer (GBC), nine $(6.6 \%)$ pediatric cases, six $(4.4 \%)$ gastrointestinal stromal tumors (GIST), six (4.4\%) adult cases of benign primary liver disease, four (2.9\%) adult cases of malignant primary liver disease, and $43(31.6 \%)$ adult cases of malignant secondary liver disease.
As for the different types of ALPPS adopted, 101 (74.3\%) cases of full ALPPS were performed, followed by 12 (8.8\%) partial ALPPS, 5 (3.7\%) radiofrequency-assisted ALPPS, $5(3.7 \%)$ tourniquet ALPPS, and 4 (2.9\%) laparoscopic ALPPS. Hybrid ALPPS, salvage ALPPS, double-in-situ ALPPS, and percutaneous microwave-assisted ALPPS were observed in $2(1.5 \%)$ cases each. One $(0.7 \%)$ case of monosegmental ALPPS was also reported (Table 1).

\section{ALPPS cases in adult patients with benign primitive liver pathologies}

Only four ALPPS cases have been reported so far for the treatment of benign primitive liver pathologies of adulthood (Table 2) (12-15). In detail, the following pathologies have 
Table 2 ALPPS cases in adult patients with benign primitive liver pathologies

\begin{tabular}{|c|c|c|c|c|}
\hline Variable & 1 & 2 & 3 & 4 \\
\hline Ref & (12) & (13) & (14) & (15) \\
\hline Year & 2018 & 2013 & 2016 & 2013 \\
\hline Affiliation & Malatya & Paris & Scandinavia & São Paulo \\
\hline Study period & 2018 & Apr 2012-May 2013 & Oct 2012-Mar 2014 & Jul 2011-Oct 2012 \\
\hline $\mathrm{N}$ & 1 & 1 & 1 & 1 \\
\hline Age (yrs) & 28 & 45 & 57 & - \\
\hline $\operatorname{Sex}(M: F)$ & M & $\mathrm{F}$ & $\mathrm{F}$ & - \\
\hline Number of lesions & 1 & - & - & - \\
\hline Primary lesion location & S4-S8 & - & - & - \\
\hline neoadjuvant treatment & no & No & - & - \\
\hline Type of liver resection & Right trisectionectomy & - & - & - \\
\hline MILS & No & Yes 1st + 2nd & - & - \\
\hline Days 1-2 stage & 8 & 7 & - & - \\
\hline FLR pre1 & - & $380 \mathrm{~mL}$ & - & - \\
\hline FLR post1 & - & $674 \mathrm{~mL}$ & - & - \\
\hline Overall survival & Alive 6 months & Alive & Alive (8.7 months) & - \\
\hline Recurrence & No & - & - & - \\
\hline
\end{tabular}

N, number; M, male; F, female; S, segment; MILS, mini-invasive liver surgery; FLR, future liver remnant; CD, Clavien-Dindo; LOS, length of stay.

been treated: echinococcus, poliadenomatosis, Caroli disease, and cystic liver disease.

As for the surgical indications, the reported case of alveolar echinococcus occupying the right lobe plus segment 4 underwent an ALPPS due to the presence of a small predicted FLR (12). Similarly, the poliadenomatosis case showed a small ratio between FLR and full liver volume (22.2\%) (13). Unfortunately, the indications for the treatment of the cases of Caroli disease and cystic liver disease were not detailed by the authors in their respective reports $(14,15)$.

The countries in which these pathologies were treated were Turkey, France, the Scandinavian area, and Brazil, respectively. The reported time interval between the first and the second ALPPS stage was 7-8 days. Detailed information on the follow-up was reported in three cases (12-14), with all the patients alive after the second stage with a follow-up ranging from 6 to 9 months. No 
information has been given on the case treated for cystic liver disease (15).

Despite the benign nature of the treated pathology, two patients experienced a Clavien-Dindo classification $\geq 3 \mathrm{a}$. In detail, both the cases reported stenosis of the left hepatic duct. The echinococcus case was treated with the placement of a percutaneous transhepatic cholangiography drainage. The patient was discharged 15 days after the ALPPS second stage, undergoing a Roux-en-Y hepaticojejunostomy six weeks after the second stage (12).

In the Caroli disease case, an initial attempt of percutaneous transhepatic cholangiography drainage failed due to non-dilated bile ducts. Therefore, after a very long length of stay (namely 261 days) in which a conservative approach was adopted with the intent to reduce the inflammatory status, the patient was re-operated with a cholangiojejunostomy (14).

\section{ALPPS cases in patients with GBC}

Twenty-seven cases of GBC infiltrating the liver parenchyma treated with ALPPS have been reported (http:// fp.amegroups.cn/cms/1395d69ef22d6ab04ec7ece0371da 2ca/hbsn-20-355-1.docx) (2,16-26). The most significant experiences reported came from the international ALPPS Registry studies, with five and six cases observed, respectively $(21,22)$. As for the single-center experiences, eight cases were reported in Germany $(16-18,23,25)$, with four cases coming from Hamburg $(18,25)$. In the reported cases, age ranged from 31 to 77 years. The extent of the tumor was specified only in three cases, with a tumor starting from the gallbladder and invading the entire right lobe and segment 4. However, although not clarified in the papers, we can postulate that in all the reported ALPPS cases, a GBC infiltrating the hepatic parenchyma or with a metastatic liver spread was present.

The decision to use a neo-adjuvant treatment was clarified in seven cases: chemotherapy was used in only two cases, while a direct surgical approach was performed in the remaining five patients. The median time interval between the first and the second ALPPS stage was 9 (ranges, 6-15) days. The decision to use an adjuvant approach was clarified only in two cases: chemotherapy was never considered as an adjuvant strategy.

Exhaustive information on survival or recurrence data was observed in only 17 cases $(17,18,20,21,22,24-26)$. Nine on $15(60 \%)$ patients died within 90 days from the second stage, therefore reporting significantly negative results.
However, five of these cases derived from an international study specifically focused on early death after ALPPS (21). Consequently, we can postulate that this factor biased the negative results.

As for the recurrence, only one case was reported (17). However, the high early ( $<90$-day) mortality should also represent a bias in the interpretation of this, apparently positive, result.

\section{ALPPS cases in adult patients with malignant primitive liver pathologies}

Only a small number of cases with malignant primitive liver pathologies other than HCC and CCC treated with ALPPS approach have been reported in adult patients (http://fp.amegroups.cn/cms/3e50f5ba5e510fa37ad19c5 0610b1139/hbsn-20-355-2.docx). In detail, we reported two cases of hemangiopericytoma, two of epithelioid hemangioendothelioma, and one of undifferentiated pleomorphic embryonal sarcoma and leiomyosarcoma (2,27-31). Three patients came from Germany, and one each from Switzerland, the United Kingdom, and Argentina. The median time interval between the first and the second ALPPS stage was 10 (ranges, 7-28) days. Only the leiomyosarcoma case has been fully described, with the patient surviving without recurrence at one year after the second stage (30).

\section{ALPPS cases in NET patients}

Forty-one patients with NET requiring the ALPPS approach have been published so far (http://fp.amegroups.

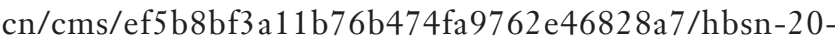
$355-3$.docx $)(22,27,28,32-39)$. In all the patients but one, the treatment was done for hepatic NET metastases. In one case, a primitive NET of the liver was reported (37). As for the site of the primitive tumor, it was clearly reported in 24 cases. In detail, the NET metastasis origin was the small bowel in ten cases, pancreas in nine, and duodenum, lung, and ovary in one case, respectively. In two cases, the primitive site of the NET tumor was unknown. The most extensive study on NET and ALPPS was an ALPPS Registry study based on 21 patients (36). The most significant single-center experience came from Argentina, with three cases (32). Other cases were reported in China, the United Kingdom, Japan, Switzerland, Germany, Chile, and Spain.

Almost in the totality of the reported cases, the NET 
liver involvement was bilobar. In the 27 patients in which the neoadjuvant approach was clearly described, we observed in detail ten cases treated with somatostatin analogs, eight with chemotherapy, eight with locoregional therapies, two with peptide receptor radiotherapy, and three cases with no neo-adjuvant therapy. In five cases, multiple approaches were contemporaneously adopted.

The primary tumor was resected before ALPPS in 23/27 $(85.2 \%)$ cases. In the four remaining patients, the site of the primary tumor was unknown (two cases) (36), the primary tumor (from the pancreas) was removed during the ALPPS second stage (one case) (33), or the primary tumor (from lungs) was not resected during the entire follow-up (one case) (36). Before ALPPS surgery, one patient also showed extra-hepatic metastatic disease, requiring a combined ALPPS plus peritonectomy for carcinosis (36). No other cases with extra-hepatic metastatic disease undergoing ALPPS have been described.

The median time interval between the first and the second ALPPS stage was 11 (ranges, 8-21) days.

As for the post-second stage complications, 9/27 (33.3\%) patients had a Dindo-Clavien grade $\geq I I I b$.

ALPPS approach showed good results for the treatment of NET, with studies reporting 1-year overall survival rates of $73-95 \%(22,36)$. As for the recurrence, 1-year disease-free survival rates ranged from $73 \%$ to $83 \%$ $(22,36)$. Unfortunately, it was impossible to clarify if the cases reported in the studies coming from the International ALPPS Registry overlapped $(22,36)$. Overall, excluding the eight cases coming from the study by Schadde et al. (22), only four on 25 (16.0\%) deaths were observed at 1, 2, 24, and 62 months, respectively.

Concerning the recurrence rates, 12 on 27 (44.4\%) cases were observed. The site of recurrence was hepatic $(n=7)$, extra-hepatic $(n=4)$, or concomitantly hepatic and extra-hepatic $(n=1)$. Patients with recurrence underwent various treatment modalities, including repeat surgery $(\mathrm{n}=5)$, locoregional therapies $(\mathrm{n}=7)$, and systemic treatments with somatostatin analogs $(n=4)$, mTOR inhibitors $(n=2)$, tyrosine kinase inhibitors $(\mathrm{n}=1)$ and $177 \mathrm{Lu}$ peptide receptor radionuclide therapy $(\mathrm{n}=1)$.

\section{ALPPS cases in GIST patients}

Six cases of GIST liver metastases treated with ALPPS have been reported (http://fp.amegroups.cn/cms/b1365fab415 40487c3171a615bc9cc4a/hbsn-20-355-4.docx) (19,40-44). The countries in which the six cases were reported were the USA, China, Brazil, Spain, Iran, and Germany. In three cases, the origin of the GIST metastasis was clarified: in detail, two cases came from the small bowel, and one from the duodenum. Patients' age ranged from 38 to 54 years. Neo-adjuvant chemotherapy with imatinib or imatininb/ sunitinib was reported in two cases $(41,44)$. The median time interval between the first and the second ALPPS stage was 10 (ranges, 8-14) days.

Only three cases were clearly described in terms of follow-up $(41,43,44)$. In all of these three cases, the liver pathology was bilobar, presenting a small FLR. DindoClavien classification $\geq \mathrm{III}$ a was observed in two of these three $(66.7 \%)$ patients. All three cases were alive at the last follow-up (ranges, 6-37 months). One patient received adjuvant chemotherapy with imatinib, being alive without recurrence 17 months after ALPPS (41). Another patient was alive without recurrence after 37 months without receiving any adjuvant medical approach (43). Only one recurrence experienced six months after the ALPPS procedure was reported (44).

\section{ALPPS cases in adult patients with other malignant secondary liver pathologies}

Forty-three ALPPS procedures have been reported for the management of malignant secondary liver pathologies other than CRLM, GBC, NET, and GIST (http://fp.amegroups. $\mathrm{cn} / \mathrm{cms} / 53 \mathrm{ab} 05 \mathrm{c} 6641 \mathrm{a} 02 \mathrm{ca} 3635536 \mathrm{e} 44 \mathrm{ae} 5 \mathrm{fc} 9 / \mathrm{hbsn}-20-355$ 5.docx) $(2,14,19,22,24,27,28,32,34,42-50)$.

A large number of primitive sites have been reported. In detail, we observed four cases of liver metastasis from sarcoma, three from melanoma, one from malignant paraganglioma, one from parotid adenoid cystic carcinoma, one from hypopharyngeal carcinoma, one from bronchial carcinoid, two from breast, two from esophageal, one from gastric, one from duodenal, two from ovarian, one from endometrial, two from uterine, two from bladder, two from clear-cell renal, and one from Wilms tumor. In 16 cases, the origin of the metastases was not clarified.

The time passed between the first and the second step was reported in only three patients, being of 7,14 , and 21 days, respectively. The follow-up was reported in eight cases: three patients died, two of whom for recurrence. The follow-up ranged from two to 40 months.

In nine cases, information on recurrence was reported: 5/9 (55.6\%) patients recurred after ALPPS. The recurred cases were two patients with esophageal metastases, two cases with metastases from cervix cancer, and one case of 
metastases from malignant paraganglioma $(32,44,46)$.

\section{ALPPS cases in pediatric patients}

Nine cases of ALPPS in pediatric patients were reported (http://fp.amegroups.cn/cms/578240bc2e664807a81a42b41 81f5444/hbsn-20-355-6.docx) (51-55). The most extensive series came from Brazil, with five cases described (55). Other countries in which pediatric ALPPS cases were reported were China, the USA, and Pakistan. One benign pathology, namely a focal nodular hyperplasia, was treated. In all the other cases, a malignant pathology was treated. In detail, six cases of hepatoblastoma, one case of hepatocarcinoma, and one case of rhabdomyosarcoma were observed. The time passed between the first and the second step was reported in only four patients, being of $8,8,14$, and 16 days, respectively (51-54). The FLR hypertrophy observed in these cases ranged from $46 \%$ to $91 \%$ (51-54).

Only in two cases, all of them presenting a hepatoblastoma, sufficient data on follow-up were reported $(53,54)$. Both the patients were alive at one and 55 months after the second stage, however one of them with an early recurrence.

\section{Discussion}

According to the most recent data reported in the International ALPPS Registry, 1,228 cases have been treated so far (http://www.alpps.net/?q=recruitment_status; 20th of February, 2020). In light of the number of uncommon liver pathology cases reported in the present study $(n=136)$, we can postulate that the uncommon liver pathology cases do not exceed ten percent of the global ALPPS activity.

The majority of articles on ALPPS have been published for the treatment of CRLM, HCC, and CCC. Consequently, reliable results are available only on these tumors.

As for the CRLM, although several concerns have been raised on the overall negative effect of ALPPS in terms of morbidity and early mortality (56), meta-analytic studies have shown that this novel surgical approach is associated with high completion rates of both the stages when compared with TSH (57). Moreover, ALPPS and TSH showed similar complication rates of the first and second surgical stage, liver failure, and 90-day mortality (57). CRLM patients reported the best overall survival rates when compared with the other ALPPS indications (58). In a study based on the International ALPPS Registry, 295 CRLM patients resected during the period 2009-2015 reported first and second stage morbidity $(\geq \mathrm{III} a)$ rates of 11.1 and $28.5 \%, 90$-day mortality rates of $7.5 \%$, and 2 -year overall and disease-free survivals of 62 and $18 \%$, respectively (59).

Concerning HCC and CCC, more contradictory results have been published, in which higher morbidity and mortality rates were observed $(6,7,60)$. As an example, a study from the International ALPPS Registry based on 35 HCC patients undergoing ALPPS reported second stage morbidity ( $\geq \mathrm{IIIb})$ rates of $26.9 \%, 90$-day mortality rates of $31.4 \%$, and 18 -month overall survival rates of $55 \%$ (6). As for the CCC, a study coming from the International ALPPS Registry based on 102 cases showed severe ( $\geq \mathrm{III}$ ) morbidity rates after the second stage of $41.4 \%$ and 90 day mortality of $21.2 \%$. The overall survival rates at 1 , 2,3 , and 5 years were $64.3 \%, 52.5 \%, 38.8 \%$ and $22.0 \%$, respectively (7).

On the opposite, there is minimal evidence on the usefulness of ALPPS in the treatment of uncommon liver pathologies, mainly due to the small series reported worldwide (Table 1). Overall, we can note that the papers focused on the ALPPS treatment of uncommon pathologies are typically case reports or case series, often with little information on clinical outcomes or short follow-up periods. Up to $30 \%$ of the studies selected in the present systematic review failed to give any clinical information apart from the pathology treated with ALPPS.

Considering the entire population of 136 patients selected in this study, we can report that the median interval between the first and the second stage was 11 days, with a range between 6 and 28 days. This datum confirms the usefulness of ALPPS over TSH in terms of hypertrophy rapidity also in pathologies other than CRLM, HCC, and CCC.

Only two pathologies presented more than ten cases collected, namely NET $(n=41 / 136 ; 30.1 \%)$ and GBC $(n=27 ; 20.0 \%)$, together reaching half of the entire cohort described in the review.

As for the NET patients, clinical data should be extracted only in a limited percentage of cases. In all the NET cases reported, the main indication for ALPPS was the bilobarity and the presence of a small FLR. The median time between the first and second ALPPS stage in NET cases was 11 days. Interestingly enough, NET represents the unique uncommon liver pathology in which a specifically focused study coming from the International ALPPS Registry has been published (36). This study, representing the most significant experience observed on bilobar NET treatment $(\mathrm{n}=21)$, reported reliable results (36). In detail, the median 
follow-up was 28 (ranges, 19-48) months, with 1 - and 2 -year disease-free survival rates of $73.2 \%$ and $41.8 \%$, respectively. As for the overall survival, 1- and 2-year rates were $95.2 \%$ and $95.2 \%$, respectively (36). Another study coming from the same international registry $(n=8)$ reported 1 -year overall and disease-free survival rates of $73 \%$ and $83 \%$, respectively (22). Unfortunately, it was not possible to clarify if overlapping data existed between these two studies, although it is highly probable $(22,36)$. In the 25 cases in which follow-up data were clearly reported, we observed 1-, 2- and 5-year rates of 92.0, 85.4, and 85.4\%.

The decision to perform an ALPPS instead of an alternative curative strategy like the liver transplantation (LT) for the treatment of NET requires further discussion. LT represents an effective strategy for the management of NET. According to recently proposed selection criteria, NET patients should be candidates for LT in the presence of (I) confirmed histology of low-grade (G1-G2) NET; (II) primary tumor drained by the portal system and removed with all extrahepatic deposits in a separate curative resection before LT consideration; (III) metastatic diffusion to $<50 \%$ of the total liver volume; (V) stable disease/response to therapies for at least six months before LT consideration; (VI) age $<60$ years (61). A systematic review evaluated the available literature comparing post-LT outcomes with those of non-transplant options: 5-year overall survival rates of transplanted patients were about the same as nontransplanted patients, but the 5 -year disease-free survival was higher in the transplanted patients (50\% vs. 34\%) (62). In a study comparing LT with resection after propensity score adjustment, a significant survival advantage was observed in the LT group (63). Unfortunately, no studies exist specifically comparing ALPPS and LT for the curative treatment of NET. Both the procedures present satisfactory survival rates, but these positive results should be balanced against high surgical morbidity and mortality rates.

Furthermore, in the case of LT patients, the long-term survival achieved exposes the patients to the long-term complications of immunosuppression (63). Therefore, the decision to consider a patient available for ALPPS or LT should be cautiously weighed case by case, taking into account several parameters like the presence of comorbidities, the aggressiveness of the tumor, its burden, and the age and performance status of the patient. To date, robust evidence showing the superiority of LT or ALPPS for the cure of NET patients is lacking.

As for the GBC, no international studies specifically focused on this pathology have been performed so far. The
27 cases reported in the current review are at risk for the presence of overlapping data in the two analyses coming from the International ALPPS Registry $(21,22)$. The main indication for ALPPS in the case of GBC was the presence of a tumor involving the right lobe and segment 4, with a contemporaneous presence of a small FLR. The median time passed between the first and the second step was 9 days, also in this case showing the rapidity of ALPPS in favoring liver hypertrophy.

Overall, the available data on the treatment of metastatic or infiltrating GBC are in line with studies based on the treatment of poorly selected CCC (64). In the present review, only 17 on 27 cases presented enough information on survival or recurrence $(17,18,20,21,22,24-26)$. The high number of nine on $15(60.0 \%)$ patients died within 90 days from the second stage. However, we should underline that such a negative result should be strongly biased from the fact that five of these cases derived from an international study exploring the post-ALPPS risk of early death (21). In general, the complete absence of studies dedicated and the high, but undoubtedly biased, risk of early death, avoid us from giving a clear answer on the efficacy of ALPPS for the treatment of advanced GBC.

Another aspect requiring a more detailed investigation is the potential impact of neoadjuvant and adjuvant treatments for the treatment of advanced GBC receiving an ALPPS approach. Unfortunately, only a limited number of studies reported the use of chemotherapy before or after ALPPS $(17,18,20,25,26)$. Recent evidence showed that locally advanced/borderline resectable GBC showed favorable response rates with neoadjuvant chemotherapy, and that satisfactory survival rates were observed in patients undergoing curative surgery after a favorable response (65). On the opposite, the existing literature does not provide clear evidence that adjuvant chemotherapy is effective in patients undergoing resection for GBC (66). According to the ASCO guidelines, adjuvant capecitabine is recommended for six months following curative resection of GBC. Researchers agree on the fact that 12-16 weeks of recovery time is required after surgery before starting adjuvant therapy (67). In light of the role potentially curative of ALPPS for the treatment of locally advanced GBC, a study aimed at clarifying the combination of chemotherapy and ALPPS in this setting is strongly needed.

Only four cases of benign pathology were reported, corresponding to only $0.3 \%$ of the total number of ALPPS reported worldwide. The only indication for ALPPS in these cases derived from the presence of an initially small 
FLR value. However, as clearly stated in several reports, ALPPS is connected with an increased risk of morbidity and mortality $(6,7,56,60)$, being too risky for the treatment of benign pathologies. In the present review, half of the reported cases showed a Clavien-Dindo complication $\geq$ IIIa. The decision to perform an ALPPS is not justified in a benign disease by the exigence of minimizing the time interval between the first and the second step for reducing the risk of tumor spread.

Consequently, in the case of benign pathologies, less aggressive approaches should be preferred for consenting the FLR hypertrophy, like the liver venous deprivation (LVD) (68). In LVD, the simultaneous embolization of hepatic and portal vein is done, inducing a more significant and faster FLR hypertrophy than after portal vein embolization (PVE) alone (69). This alternative FLR increasing technique should represent a future promising and less invasive procedure respect to the, evidently more aggressive, ALPPS approach, mainly if a non-tumoral pathology is managed.

Nine pediatric cases have also been described so far, with four different treated pathologies, the most commonly observed of whom was the hepatoblastoma (six cases). We observed with interest the impact of ALPPS in pediatric patients. We can postulate that the regeneration capacity is higher in pediatric liver respect to the adult one. However, specific guidelines investigating a different interval between first and second stage ALLPS in children does not exist. According to the experiences reported, a time interval ranging from 8 to 16 days was described, with a FLR hypertrophy ranging $46-91 \%(51-54)$. These data are in line with the ones reported in the adult experiences. Therefore, according to the evidence reported in the literature, it is not possible to obtain firm conclusions on the possible faster-growing process in children. The ALPPS approach in childhood should follow the same temporal rules of adulthood.

As for the secondary liver malignant pathologies, more than 15 different primitive sites have been reported, with up to $40 \%$ of cases without a clear description of the primitive tumor. Overall, we observed a limited number of cases, followed by a high heterogeneity of the data, and an overall paucity of information. Therefore, a note of caution should be given on the role of ALPPS in this setting. In the case of malignant pathologies, radioembolization has been proposed as an emerging radiological approach, able to induce contralateral liver hypertrophy with simultaneous ipsilateral control of tumor growth $(70,71)$. Respect to PVE, radioembolization presents the great benefit of contextually treat the tumor. Respect to ALPPS, this procedure consents to obtain noninvasive hypertrophy of the FLR, therefore minimizing the risk of morbidity and mortality. The principal limit of radioembolization is the slower rate of hypertrophy respect to that achieved by the other methods. As an example, a systematic review based on 312 patients showed liver hypertrophy ranging from $26 \%$ to $47 \%$ with time from treatment to hypertrophy ranging from 44 days to 9 months (71). As a consequence, the decision to adopt an aggressive approach like the ALPPS in these patients should be considered in very well selected cases in which other approaches are not usable due to technical challenges or for the presence of tumor behaviors of aggressiveness.

\section{Conclusions}

Less than ten percent of ALPPS procedures are performed for the treatment of uncommon liver pathologies. Among them, NET and GBC are the unique pathologies showing acceptable numerosity. ALPPS for bilobar NET metastases appears to be a safe procedure, with satisfactory longterm results. Indication for ALPPS should be accurately selected in NET cases, considering the existence of a valid alternative curative therapy like the liver transplantation.

On the opposite, the results observed for the treatment of GBC are poor. However, these data should be considered with caution due to possible selection biases present in the analyses reported.

There is not a clear justification for treating benign pathologies with ALPPS, mainly considering the existence of less invasive strategies for resolving the problem of small FLR. No definitive response should be given for all the other classes of pathologies described. Multicenter registry studies are needed for all of these uncommon pathologies, with the primary intent to clarify the potentially beneficial effect of ALPPS for their treatment. Due to the high mortality and morbidity rates of ALPPS, this invasive surgical procedure should be performed only in well-selected cases and in very high-specialized centers, where interventional radiologic procedures like PVE, radioembolization, and LVD are routinely adopted as potential alternative strategies for favoring the FLR hypertrophy.

\section{Acknowledgments}

Funding: None. 


\section{Footnote}

Conflicts of Interest: All authors have completed the ICMJE uniform disclosure form (available at https://hbsn. amegroups.com/article/view/10.21037/hbsn-20-355/coif). The authors have no conflicts of interest to declare.

Ethical Statement: The authors are accountable for all aspects of the work in ensuring that questions related to the accuracy or integrity of any part of the work are appropriately investigated and resolved.

Open Access Statement: This is an Open Access article distributed in accordance with the Creative Commons Attribution-NonCommercial-NoDerivs 4.0 International License (CC BY-NC-ND 4.0), which permits the noncommercial replication and distribution of the article with the strict proviso that no changes or edits are made and the original work is properly cited (including links to both the formal publication through the relevant DOI and the license). See: https://creativecommons.org/licenses/by-nc-nd/4.0/.

\section{References}

1. de Santibañes E, Clavien PA. Playing Play-Doh to prevent postoperative liver failure: the "ALPPS" approach. Ann Surg 2012;255:415-7.

2. Schnitzbauer AA, Lang SA, Goessmann H, et al. Right portal vein ligation combined with in situ splitting induces rapid left lateral liver lobe hypertrophy enabling 2 -staged extended right hepatic resection in small-for-size settings. Ann Surg 2012;255:405-14.

3. Schadde E, Ardiles V, Slankamenac K, et al. ALPPS offers a better chance of complete resection in patients with primarily unresectable liver tumors compared with conventional-staged hepatectomies: results of a multicenter analysis. World J Surg 2014;38:1510-9.

4. Cai YL, Song PP, Tang W, et al. An updated systematic review of the evolution of ALPPS and evaluation of its advantages and disadvantages in accordance with current evidence. Medicine (Baltimore) 2016;95:e3941.

5. Aldrighetti L, Ratti F, Cillo U, et al;Italian Group of Minimally Invasive Liver Surgery (I GO MILS). Diffusion, outcomes and implementation of minimally invasive liver surgery: a snapshot from the I Go MILS (Italian Group of Minimally Invasive Liver Surgery) Registry. Updates Surg 2017;69:271-83.

6. D'Haese JG, Neumann J, Weniger M, et al. Should
ALPPS be Used for Liver Resection in Intermediate-Stage HCC? Ann Surg Oncol 2016;23:1335-43.

7. Li J, Moustafa M, Linecker M, et al. ALPPS for Locally Advanced Intrahepatic Cholangiocarcinoma: Did Aggressive Surgery Lead to the Oncological Benefit? An International Multi-center Study. Ann Surg Oncol 2020;27:1372-84.

8. Hasselgren K, Røsok BI, Larsen PN, et al. ALPPS Improves Survival Compared With TSH in Patients Affected of CRLM: Survival Analysis From the Randomized Controlled Trial LIGRO. Ann Surg 2019. [Epub ahead of print].

9. Huiskens J, Schadde E, Lang H, et al. Avoiding postoperative mortality after ALPPS-development of a tumor-specific risk score for colorectal liver metastases. HPB (Oxford) 2019;21:898-905.

10. Hutton B, Salanti G, Caldwell DM, et al. The PRISMA extension statement for reporting of systematic reviews incorporating network meta-analyses of health care interventions: checklist and explanations. Ann Intern Med 2015;162:777-84.

11. Stang A. Critical evaluation of the Newcastle-Ottawa scale for the assessment of the quality of nonrandomized studies in metaanalyses. Eur J Epidemiol 2010;25:603-5.

12. Akbulut S, Cicek E, Kolu M, et al. Associating liver partition and portal vein ligation for staged hepatectomy for extensive alveolar echinococcosis: First case report in the literature. World J Gastrointest Surg 2018;10:1-5.

13. Brustia R, Scatton O, Perdigao F, et al. Vessel identifications tags for open or laparoscopic associating liver partition and portal vein ligation for staged hepatectomy. J Am Coll Surg 2013;217:e51-5.

14. Røsok BI, Björnsson B, Sparrelid E, et al. Scandinavian multicenter study on the safety and feasibility of the associating liver partition and portal vein ligation for staged hepatectomy procedure. Surgery 2016;159:1279-86.

15. Torres OJ, Fernandes Ede S, Oliveira CV, et al. Associating liver partition and portal vein ligation for staged hepatectomy (ALPPS): the Brazilian experience. Arq Bras Cir Dig 2013;26:40-3.

16. Knoefel WT, Gabor I, Rehders A, et al. In situ liver transection with portal vein ligation for rapid growth of the future liver remnant in two-stage liver resection. $\mathrm{Br} \mathrm{J}$ Surg 2013;100:388-94.

17. Kremer M, Manzini G, Hristov B, et al. Impact of Neoadjuvant Chemotherapy on Hypertrophy of the Future Liver Remnant after Associating Liver Partition and Portal Vein Ligation for Staged Hepatectomy. J Am 
Coll Surg 2015;221:717-28.e1.

18. Li J, Kantas A, Ittrich H, et al. Avoid "All-Touch" by Hybrid ALPPS to Achieve Oncological Efficacy. Ann Surg 2016;263:e6-7.

19. Machado MA, Makdissi FF, Surjan RC, et al. Transition from open to laparoscopic ALPPS for patients with very small FLR: the initial experience. HPB (Oxford) 2017;19:59-66.

20. Ratti F, Cipriani F, Gagliano A, et al. Defining indications to ALPPS procedure: technical aspects and open issues. Updates Surg 2014;66:41-9.

21. Schadde E, Raptis DA, Schnitzbauer AA, et al. Prediction of Mortality After ALPPS Stage-1: An Analysis of 320 Patients From the International ALPPS Registry. Ann Surg 2015;262:780-5.

22. Schadde E, Ardiles V, Robles-Campos R, et al;ALPPS Registry Group. Early survival and safety of ALPPS: first report of the International ALPPS Registry. Ann Surg 2014;260:829-36.

23. Stockmann M, Bednarsch J, Malinowski M, et al. Functional considerations in ALPPS - consequences for clinical management. HPB (Oxford) 2017;19:1016-25.

24. Truant S, Scatton O, Dokmak S, et al. e-HPBchir Study Group from the Association de Chirurgie HépatoBiliaire et de Transplantation (ACHBT). Associating liver partition and portal vein ligation for staged hepatectomy (ALPPS): impact of the inter-stages course on morbimortality and implications for management. Eur J Surg Oncol 2015;41:674-82.

25. Tsui TY, Heumann A, Vashist YK, et al. How we do it: double in situ split for staged mesohepatectomy in patients with advanced gall bladder cancer and marginal future liver remnant. Langenbecks Arch Surg 2016;401:565-71.

26. Zhai B, Jin X, Wang R, et al. Applying negative pressure wound therapy in associating liver partition and portal vein ligation for staged hepatectomy: A case report. Exp Ther Med 2017;14:642-6.

27. Petrowsky H, Györi G, de Oliveira M, et al. Is partialALPPS safer than ALPPS? A single-center experience. Ann Surg 2015;261:e90-2.

28. Stavrou GA, Donati M, Fard-Aghaie MH, et al. Did the International ALPPS Meeting 2015 Have an Impact on Daily Practice? The Hamburg Barmbek Experience of 58 Cases. Visc Med 2017;33:456-61.

29. Schepelew D, Reese T, Horling K, et al. Undifferentiated embryonal sarcoma of the liver treated with associating liver partition and portal vein ligation for staged hepatectomy in a young adult: A case report. Int J Surg
Case Rep 2020;66:221-7.

30. Schlegel A, Sakuraoka Y, Kalisvaart M, et al. Future liver remnant portal or hepatic vein reconstruction at stage I of ALPPS-How far can we push the boundaries? J Surg Oncol 2019;120:654-60.

31. Schelotto PB, Gondolesi G. Laparoscopy in ALPPS Procedure: When We Can Do It? Ann Surg 2017;265: e30-e31.

32. Alvarez FA, Ardiles V, de Santibañes M, et al. Associating liver partition and portal vein ligation for staged hepatectomy offers high oncological feasibility with adequate patient safety: a prospective study at a single center. Ann Surg 2015;261:723-32.

33. Ji R, Zuo S, Qiu S, et al. Combined associating liver partition and portal vein ligation for staged hepatectomy (ALPPS) followed by left trisectionectomy and Whipple operation for PNET. Gland Surg 2018;7:47-53.

34. Jiao LR, Fajardo Puerta AB, Gall TMH, et al. Rapid Induction of Liver Regeneration for Major Hepatectomy (REBIRTH): A Randomized Controlled Trial of Portal Vein Embolisation versus ALPPS Assisted with Radiofrequency. Cancers (Basel) 2019;11:302.

35. Kawaguchi D, Hiroshima Y, Matsuo K, et al. Parenchymal Congestion Is Important for Rapid Regeneration of the Future Liver Remnant Following the ALPPS Procedure. Anticancer Res 2016;36:4731-8.

36. Linecker M, Kambakamba P, Raptis DA, et al. ALPPS in neuroendocrine liver metastases not amenable for conventional resection - lessons learned from an interim analysis of the International ALPPS Registry. HPB (Oxford) 2020;22:537-44.

37. Qu C, Qu LL, Zhu CZ, et al. Treatment of primary hepatic neuroendocrine tumors with associating liver partition and portal vein ligation for staged hepatectomy (ALPPS): A case report and literature review. Medicine (Baltimore) 2018;97:e12408.

38. Uribe M, Uribe-Echevarría S, Mandiola C, et al. Insight on ALPPS - Associating Liver Partition and Portal Vein Ligation for Staged Hepatectomy - mechanisms: activation of mTOR pathway. HPB (Oxford) 2018;20:729-38.

39. Vicente E, Quijano Y, Ielpo B, et al. Is "small for size syndrome" a relatively new complication after the ALPPS procedure? Updates Surg 2015;67:273-8.

40. Croome KP, Hernandez-Alejandro R, Parker M, et al. Is the liver kinetic growth rate in ALPPS unprecedented when compared with PVE and living donor liver transplant? A multicentre analysis. HPB (Oxford) 2015;17:477-84. 
41. Liu J, Zhang C, Hong D, et al Percutaneous microwave ablation liver partition and portal vein embolization for planned hepatectomy due to large gastrointestinal stromal tumor metastases: A case report. Medicine (Baltimore) 2017;96:e8271.

42. Robles R, Parrilla P, López-Conesa A, et al. Tourniquet modification of the associating liver partition and portal ligation for staged hepatectomy procedure. Br J Surg 2014;101:1129-34.

43. Sanei B, Sheikhbahaei S, Sanei MH, et al. Associating liver partition and portal vein ligation for staged hepatectomy: A surgical technique for liver resections. J Res Med Sci 2017;22:52.

44. Troja A, Khatib-Chahidi K, El-Sourani N, et al. ALPPS and similar resection procedures in treating extensive hepatic metastases: our own experiences and critical discussion. Int J Surg 2014;12:1020-2.

45. Enne M, Schadde E, Björnsson B, et al. ALPPS Registry Group. ALPPS as a salvage procedure after insufficient future liver remnant hypertrophy following portal vein occlusion. HPB (Oxford) 2017;19:1126-9.

46. Lang SA, Loss M, Benseler V, et al. Long-term results after in-situ split (ISS) liver resection. Langenbecks Arch Surg 2015;400:361-9.

47. Lunardi A, Cervelli R, Volterrani D, et al. Feasibility of Percutaneous Intrahepatic Split by Microwave Ablation (PISA) After Portal Vein Embolization for Hypertrophy of Future Liver Remnant: The Radiological Stage-1 ALPPS. Cardiovasc Intervent Radiol 2018;41:789-98.

48. Olthof PB, Tomassini F, Huespe PE, et al. Hepatobiliary scintigraphy to evaluate liver function in associating liver partition and portal vein ligation for staged hepatectomy: Liver volume overestimates liver function. Surgery 2017;162:775-83.

49. Tomassini F, D'Asseler Y, Giglio MC, et al. Hemodynamic changes in ALPPS influence liver regeneration and function: results from a prospective study. HPB (Oxford) 2019;21:557-65.

50. Ulmer TF, de Jong C, Andert A, et al. ALPPS Procedure in Insufficient Hypertrophy After Portal Vein Embolization (PVE). World J Surg 2017;41:250-7.

51. Chan A, Chung PH, Poon RT. Little girl who conquered the "ALPPS". World J Gastroenterol 2014;20:10208-11.

52. Hong JC, Kim J, Browning M, et al. Modified Associating Liver Partition and Portal Vein Ligation for Staged Hepatectomy for Hepatoblastoma in a Small Infant: How Far Can We Push the Envelope? Ann Surg 2017;266:e16-7.

53. Qazi AQ, Syed AA, Khan AW, et al. Early multifocal recurrence of hepatoblastoma in the residual liver after R0 liver resection with ALPPS procedure: a case report. Ann Transl Med 2016;4:375.

54. Ruiz Figueroa EF, Fernández-Placencia RM, Berrospi Espinoza FE, et al. Monosegmental ALPPS: a long-term survival alternative to liver transplant in PRETEXT IV hepatoblastoma. J Surg Case Rep 2019;2019:rjz144.

55. Wiederkehr JC, Avilla SG, Mattos E, et al. Associating liver partition with portal vein ligation and staged hepatectomy (ALPPS) for the treatment of liver tumors in children. J Pediatr Surg 2015;50:1227-31.

56. Adam R, Imai K, Castro Benitez C, et al. Outcome after associating liver partition and portal vein ligation for staged hepatectomy and conventional two-stage hepatectomy for colorectal liver metastases. Br J Surg 2016;103:1521-29.

57. Shen YN, Guo CX, Wang LY, et al. Associating liver partition and portal vein ligation versus 2-stage hepatectomy: A meta-analysis. Medicine (Baltimore) 2018;97:e12082.

58. Linecker M, Stavrou GA, Oldhafer KJ, et al. The ALPPS Risk Score: Avoiding Futile Use of ALPPS. Ann Surg 2016;264:763-71.

59. Olthof PB, Huiskens J, Wicherts DA, et al. Survival after associating liver partition and portal vein ligation for staged hepatectomy (ALPPS) for advanced colorectal liver metastases: A case-matched comparison with palliative systemic therapy. Surgery 2017;161:909-19.

60. Tustumi F, Ernani L, Coelho FF, et al. Preoperative strategies to improve resectability for hepatocellular carcinoma: a systematic review and meta-analysis. HPB (Oxford) 2018;20:1109-18.

61. Mazzaferro V, Pulvirenti A, Coppa J. Neuroendocrine tumors metastatic to the liver: how to select patients for liver transplantation? J Hepatol 2007;47:460-6.

62. Fan ST, Le Treut YP, Mazzaferro V, et al. Liver transplantation for neuroendocrine tumour liver metastases. HPB (Oxford) 2015;17:23-8.

63. Mazzaferro V, Sposito C, Coppa J, et al. The long-term benefit of liver transplantation for hepatic metastases from neuroendocrine tumors. Am J Transplant 2016;16:2892-902.

64. Olthof PB, Coelen RJS, Wiggers JK, et al. High mortality after ALPPS for perihilar cholangiocarcinoma: case-control analysis including the first series from the international ALPPS registry. HPB (Oxford) 2017;19:381-7.

65. Chaudhari VA, Ostwal V, Patkar S, et al. Outcome of neoadjuvant chemotherapy in "locally advanced/borderline 
resectable" gallbladder cancer: the need to define indications. HPB (Oxford) 2018;20:841-7.

66. Manterola C, Duque G, Grande L, et al. A systematic review of the effectiveness of adjuvant therapy for patients with gallbladder cancer. HPB 2019;21:1427-35.

67. Shroff RT, Kennedy EB, Bachini M, et al. Adjuvant Therapy for Resected Biliary Tract Cancer: ASCO Clinical Practice Guideline. J Clin Oncol 2019;37:1015-27.

68. Panaro F, Giannone F, Riviere B, et al. Perioperative impact of liver venous deprivation compared with portal venous embolization in patients undergoing right hepatectomy: preliminary results from the pioneer center. Hepatobiliary Surg Nutr 2019;8:329-37.

Cite this article as: Lai Q, Mennini G, Larghi Laureiro Z, Rossi M. Uncommon indications for associating liver partition and portal vein ligation for staged hepatectomy: a systematic review. HepatoBiliary Surg Nutr 2021;10(2):210-225. doi: 10.21037/hbsn-20-355
69. Kobayashi K, Yamaguchi T, Denys A, et al. Liver venous deprivation compared to portal vein embolization to induce hypertrophy of the future liver remnant before major hepatectomy: A single center experience. Surgery 2020;167:917-23.

70. Birgin E, Rasbach E, Seyfried S, et al. Contralateral Liver Hypertrophy and Oncological Outcome Following Radioembolization with 90Y-Microspheres: A Systematic Review. Cancers (Basel) 2020;12:E294.

71. Teo JY, Allen JC Jr, Ng DC, et al. A systematic review of contralateral liver lobe hypertrophy after unilobar selective internal radiation therapy with Y90. HPB (Oxford) 2016;18:7-12. 